Modern Physics Letters A

Vol. 28, No. 40 (2013) 1399001 (12 pages)

(C) World Scientific Publishing Company

DOI: 10.1142/S0217732313990010

\title{
AUTHOR INDEX Volume 28
}

Aarnink, T., see van der Graaf

Aarts, A., see van der Graaf

Abbas, G., Ananthanarayan, B. \& Caprini, I., Determination of the strong coupling from hadronic tau decays using renormalization group summed perturbation theory

Abbyazov, R. R. \& Chervon, S. V., Unified dark matter and dark energy description in a chiral cosmological model

Accardi, A., Large- $x$ connections of nuclear and high-energy physics

Adak, M., see Sert

Agop, M., see Mazilu

Aizawa, N. \& Roy, P., Coherent state of the extended Scarf I potential and some of its properties

Ajaltouni, Z. J., see Di Salvo

Ajaz, M., Suleymanov, M. K., Khan, K. H., Zaman, A., Younis, H. \& Rahman, A., Study of some characteristics of protons using interactions of light nuclei

Alavi, S. A., see Pahlavani

Alekhin, S., Blümlein, J. \& Moch, S.-O., Determination of $\alpha_{s}$ and $m_{c}$ in deep-inelastic scattering

Allal, N. H., see Oudih

Amorim, R. G. G., see Ulhoa

Ananthanarayan, B., see Abbas

Antipin, O., Sannino, F. \& Tuominen,

K., The mirage of the Fermi scale

Arık, M. see Şengör

Arraut, I., About the propagation of the gravitational waves in an asymptotically de Sitter space: Comparing two points of view

Artamonov, A., Charmonia production at $\mathrm{LHCB}$

Artun, O., see Aytekin
28 (2013) 1340021

28 (2013) 1340021

28(2013) 1350024

28(2013) 1330032

28(2013) 1350190

28(2013) 1350126

28 (2013) 1350123

28 (2013) 1350048

28 (2013) 1350175

28 (2013) 1350065

28 (2013) 1360018

28 (2013) 1350134

28(2013) 1350039

28 (2013) 1360004

28 (2013) 1350140

28(2013) 1350095

28 (2013) 1350019

28 (2013) 1330037

28 (2013) 1350007
Asselmeyer-Maluga, T. \& Król, J., Decoherence in quantum cosmology and the cosmological constant Aygun, M., Boztosun, I. \& Rusek, K., Parametrized form of the dynamic polarization potential for the ${ }^{6} \mathrm{He}+{ }^{208} \mathrm{~Pb}$ interaction

Aytekin, H. \& Artun, O., An investigation of the nuclear structures of even-even neutron-rich $\mathrm{Sr}, \mathrm{Zr}$ and Mo isotopes

Babaro, J. P. \& Giribet, G., On the description of surface operators in $\mathcal{N}=2^{*} \mathrm{SYM}$

Bagchi, B., Ghose Choudhury, A. \& Guha, P., Comments on the structural features of the PaisUhlenbeck oscillator

Bai, N., Gao, Y.-H., Qi, B.-G. \& $\mathrm{Xu}, \mathrm{X}$. -B., Quasinormal frequencies of black branes with hyperscaling violation

Balachandran, A. P., Kürkçüoğlu, S. \& de Queiroz, A. R., Spontaneous breaking of Lorentz symmetry and vertex operators for vortices

Barducci, A. \& Giachetti, R., Effective action for fermions with anomalous magnetic moment from Foldy-Wouthuysen transformation

Bartalini, P., Multi-particle production of hadrons

Bayram, T. \& Yilmaz, A. H., Table of ground state properties of nuclei in the RMF model

Bello, D. S. S., see van der Graaf

Belloni, F., Gunsing, F. \& Papaevangelou, T., Micromegas for neutron detection and imaging

Beltrán Almeida, J. P., Rodríguez, Y. \& Valenzuela-Toledo, C. A.,
$28(2013) 1350158$

28(2013) 1350112

28 (2013) 1350007

28 (2013) 1330003

28 (2013) 1375001

28 (2013) 1350145

28 (2013) 1350028

28 (2013) 1350029

28 (2013) 1330010

28 (2013) 1350068

28 (2013) 1340021

28 (2013) 1340023 
The Suyama-Yamaguchi consistency relation in the presence of vector fields

Benchikha, A. \& Chetouani, L., Energy-dependent potential and normalization of wave function

Benhamouda, N., see Oudih

Bennai, M., see Zarrouki

Benzair, H., Merad, M. \& Boudjedaa, T., Noncommutative path integral for spinless relativistic equation in the two-component theory

Berbee, E., see van der Graaf

Berkien, A., see van der Graaf

Bezerra, V. B., see de A. Marques

Bhattacharjee, A. \& Deshamukhya, A., Geometric tachyon and warm inflation

Bian, J.-G., see Tao

Birrell, J., Yang, C.-T., Chen, P. \& Rafelski, J., Fugacity and reheating of primordial neutrinos

Blümlein, J., see Alekhin

Bodenstein, S., Precise determinations of the charm and bottom quark masses

Bodenstein, S., The hadronic contribution to the muon magnetic anomalous moment

Bosma, M., see van der Graaf

Bouchareb, A., see Ydri

Boudjedaa, T., see Benzair

Bozkurt, K., see E. Yüksel

Boztosun, I., see Aygun

Braghin, F. L., Fierz transformation for $\mathrm{SU}(2)$ and $\mathrm{SU}(3)$ eighth-order fermion interactions

Brevik, I., Timoshkin, A. V. \& Rabochaya, Y., Little rip and pseudo rip phenomena from coupled dark energy

Brito, L. C. T., see Felipe

Brüning, O. \& Klein, M., The large hadron electron collider

Butenschoen, M. \& Kniehl, B. A., Next-to-leading order tests of non-relativistic-QCD factorization with $J / \psi$ yield and polarization

Cabral, L. A., see Felipe

Çakir, I. T., Çakir, O., Senol, A. \& Tasci, A. T., Probing anomalous $\mathrm{HZZ}$ couplings at the $\mathrm{LHeC}$

Çakir, O., see Çakir

Cai, R., see Zhang

Calderón Martín, A. J. \& Sánchez-

Delgado, J. M., Weight modules over split Lie algebras

28 (2013) 1350012

28 (2013) 1350079

28 (2013) 1350134

28 (2013) 1350062

28 (2013) 1350144

28 (2013) 1340021

28 (2013) 1340021

28 (2013) 1350137

28 (2013) 1350036

28 (2013) 1350081

28 (2013) 1350188

28 (2013) 1360018

28 (2013) 1360020

28 (2013) 1360021

28 (2013) 1340021

28 (2013) 1350166

28 (2013) 1350144

28 (2013) 1350177

28 (2013) 1350112

28 (2013) 1350023

28 (2013) 1350172

28 (2013) 1350078

28 (2013) 1330011

28 (2013) 1350027

28 (2013) 1350078

28 (2013) 1350142

28 (2013) 1350142

28 (2013) 1350009
Campbell, M., see van der Graaf

Capozziello, S., Fabbri, L. \& Vignolo, S., Weak forces and neutrino oscillations under the standards of hybrid gravity with torsion

Caprini, I., Strong coupling from the tau hadronic width by non-power QCD perturbation theory

Caprini, I., see Abbas

Carr, B. J., Black holes, the generalized uncertainty principle and higher dimensions

Cerri, A., Recent heavy flavor results from the ATLAS experiment

Chakrabortty, J., Das, M. \& Mohanty, S., Constraints on TeV scale Majorana neutrino phenomenology from the vacuum stability of the Higgs

Chakraborty, S. \& Dey, P., WessZumino-Witten model for Galilean conformal algebra

Chefdeville, M., see van der Graaf

Chen, B., see Zhao

Chen, C.-C., Chen, P., Hu, C.-Y. \& Lai, K.-C., Distinguishability of neutrino flavors through their different shower characteristics

Chen, C.-C., see $\mathrm{Hu}$

Chen, G.-M., see Tao

Chen, H.-S., see Tao

Chen, J.-B., see Sun

Chen, P. \& Wang, C.-H., Quantum corrections to entropic gravity

Chen, P., see Birrell

Chen, P., see Chen

Chen, P., see Hu

Chen, Y., see Tao

Chervon, S. V., see Abbyazov

Chetouani, L., see Benchikha

Chimento, L. P., Forte, M. \& Richarte, M. G., Self-interacting holographic dark energy

Chimento, L. P., Richarte, M. G. \& Sánchez, I. E., Form invariance symmetry generates a large set of FRW cosmologies

Ching, C.-L. \& Parwani, R. R., Effect of maximum momentum on quantum mechanical scattering and bound states

Cho, G.-C., Nomura, D. \& Ohno, Y., Constraints on radion in a warped
28 (2013) 1350008

28 (2013) 1340021

28 (2013) 1350155

28 (2013) 1360003

28 (2013) 1360004

28 (2013) 1340011

28 (2013) 1330021

28 (2013) 1350032

28 (2013) 1350176

28 (2013) 1340021

28 (2013) 1350173

28 (2013) 1340009 28 (2013) 1340006 28 (2013) 1350081

28 (2013) 1350081

28 (2013) 1350151

28 (2013) 1340010

28 (2013) 1350188

28 (2013) 1340009

28 (2013) 1340006

28 (2013) 1350081

28 (2013) 1350024

28(2013) 1350079

28 (2013) 1250235

28 (2013) 1250236

28 (2013) 1350061 
extra dimension model from Higgs boson searches at the LHC

Choi, J., Kim, Y.-W. \& Park, Y.-J., The geodesic motion near hypersurfaces in the warped products spacetime

Choudhury, D. K., see Jahan

Chung, W. S. \& Jung, M., Some new properties concerning the $q$-deformed calculus and the $q$-deformation of the diffusion equation

Chung, W. S., On the representation of $A_{\kappa}(d)$ algebra

Chung, W. S., On the representation of the Wigner algebra and Wigner deformation of the fermion algebra

Cirilo-Lombardo, D. J., see Prudêncio

Cleaver, G., see Moore

Colas, P., see van der Graaf

Colijn, A.-P., see van der Graaf

Cooper-Sarkar, A. M., Determination of charm quark mass and $\alpha_{s}\left(M_{z}\right)$ from HERA data

Cornell, A. S., Deandrea, A., Liu, L.-X. \& Tarhini, A., Renormalization running of masses and mixings in UED models

Cornwall, J. M., Positivity violations in QCD

Cotăescu, I. I., Covariant representations of the de Sitter isometry group

Cotăescu, I. I., Pascu, G. \& Dregoesc, F. A., On the rest and flat limits of the scalar modes on the de Sitter spacetime

Crewther, R. J. \& Tunstall, L. C., Infrared fixed point in the strong running coupling: Unraveling the $\Delta I=1 / 2$ puzzle in $K$-decays

Cristofano, G., Maiella, G. \& Stornaiolo, C., Quantization of black holes entropy and its cosmological consequences

Cui, L.-X. \& Wu, Y.-L., Thermal mass spectra of scalar and pseudoscalar mesons in IR-improved soft-wall AdS/QCD model with finite chemical potential

da Silva, A. J., see Ferrari

Dafni, T., see Irastorza

Darabi, F., see Zeynali
28 (2013) 1350148

28 (2013) 1350143

28 (2013) 1350086

28 (2013) 1350100

28(2013) 1350115

28 (2013) 1350074

28 (2013) 1350058

28 (2013) 1350055

28 (2013) 1340021

28 (2013) 1340021

28 (2013) 1360017

28 (2013) 1330007

28 (2013) 1330035

28 (2013) 1350033

28 (2013) 1350160

28 (2013) 1360010

28 (2013) 1350066

28 (2013) 1350132

28 (2013) 1350052

28(2013) 1340026

28 (2013) 1350047
Dariescu, C., see Dariescu

Dariescu, M.-A. \& Dariescu, C., On the Mathieu equation describing ultra-relativistic fermions evolving in a time-harmonic electric field parallel to a static magnetic induction

Das, D., see Kisslinger

Das, D., see Kisslinger

Das, M., see Chakrabortty

Daszkiewicz, M., Equivalent forces in Newton equation from twist deformations and non-inertial coordinate frames

Datta, A., see Rashed

de A. Marques, G., Bezerra, V. B. \& Dong, S.-H., Solution of the Dirac equation with position-dependent mass in a Coulomb and scalar fields in a conical spacetime

de Araujo, J. C. N., see Evangelista

de Groot, N., see van der Graaf

de Nooij, L., see van der Graaf

de Queiroz, A. R., see Balachandran

Deandrea, A., see Cornell

Deepthi, K. N., see Gollu

Demir, S., see Tanışlı

Deng, Y., see Sun

Denig, A., Review of $R$ measurements

Deriglazov, A. A., Variational problem for the Frenkel and the Bargmann-Michel-Telegdi (BMT) equations

Deshamukhya, A., see Bhattacharjee Dey, P., see Chakraborty

Di Salvo, E. \& Ajaltouni, Z. J., Baryon polarization, phases of amplitudes and time-reversal odd observables

Diakonos, F. K., see Mazilu

Disconzi, M. M., A note on quantization in the presence of gravitational shock waves

Dominguez, C. A., Introduction to QCD sum rules

Dong, S.-H., see de A. Marques

Dregoesc, F. A., see Cotăescu

Du, Y. J., see Gao

Ermolaev, B. I., Greco, M. \& Troyan, S. I., On the frozen QCD coupling Escobar-Aguilar, E. S., see Romero

Eser, E., see Gokbulut

Evangelista, E. F. D. \& de Araujo, J. C. N., A new method to calcu-
28 (2013) 1350157

28 (2013) 1350157

28 (2013) 1350067

28 (2013) 1350120

28 (2013) 1350032

28 (2013) 1350154

28 (2013) 1330030

28 (2013) 1350137

28(2013) 1350174

28 (2013) 1340021

28 (2013) 1340021

28 (2013) 1350028

28 (2013) 1330007

28 (2013) 1350131

28(2013) 1350026

28 (2013) 1350114

28 (2013) 1360008

28 (2013) 1250234

28 (2013) 1350036

28 (2013) 1350176

28(2013) 1350048

28(2013) 1350126

28 (2013) 1350111

28 (2013) 1360002

28 (2013) 1350137

28 (2013) 1350160

28 (2013) 1350138

28 (2013) 1360005

28 (2013) 1350004

28 (2013) 1350108 
late the stochastic background of gravitational waves generated by compact binaries

Fabbri, L., see Capozziello

Fabris, J. C., Piattella, O. F., Velten, H. E. S., Salako, I. G. \& Tossa, J., A note on acoustic black holes in neo-Newtonian theory

Faizal, M., Noncommutative quantum gravity

Fan, J.-W., see Tao

Feliciello, A., The last results from the FINUDA experiment

Felipe, J. C. C., Cabral, L. A., Brito, L. C. T., Sampaio, M. \& Nemes, M. C., Ambiguities in the gravitational correction of quantum electrodynamics

Fellah, M., see Oudih

Feng, T.-F., see Sun

Feng, T.-F., see Sun

Feng, T.-F., see Zhao

Fernando, S., Nariai black holes with quintessence

Ferrari, A. F., Girotti, H. O., Gomes, M., Yu. Petrov, A. \& da Silva, A. J., Hor̆ava-Lifshitz modifications of the Casimir effect

Ferrer-Ribas, E., see Irastorza

Ferretti, G. \& Karateev, D., Chiral extensions of the MSSM

Fornaini, A., see van der Graaf

Forte, M., see Chimento

Frampton, P. H., Remarks on the cosmological constant

Frampton, P. H. \& Ludwick, K. J., Cyclic cosmology from the little rip

Franceschini, R., On the factorization of the scattering of $W$ bosons

Fransen, M., see van der Graaf

Fried, H. M., A new, analytic, nonperturbative, gauge-invariant, realistic formulation of QCD

Fritzsch, H., Composite weak bosons and dark matter

Fritzsch, H., History of QCD

Frolov, I. V., Martynov, M. V. \& Smirnov, A. D., The chiral color symmetry of quarks and $G^{\prime}$-boson contributions to charge asymmetry in $t \bar{t}$-production at the $\mathrm{LHC}$ and Tevatron

Fukuyama, T. \& Nishiura, H., Universality of quark-lepton mass matrix

Fukuyama, T. \& Nishiura, H., Hierarchical masses and universal mix-

28 (2013) 1350174

28 (2013) 1350155

28 (2013) 1350169

28 (2013) 1350034

28 (2013) 1350081

28 (2013) 1330029

28 (2013) 1350078

28 (2013) 1350134

28 (2013) 1350060

28 (2013) 1350151

28 (2013) 1350173

28 (2013) 1350189

28 (2013) 1350052

28 (2013) 1340026

28 (2013) 1350025

28 (2013) 1340021

28 (2013) 1250235

28 (2013) 1350001

28 (2013) 1350125

28 (2013) 1330008

28 (2013) 1340021

28 (2013) 1230045

28 (2013) 1350106

28 (2013) 1360001

28 (2013) 1350017 ing in quark-lepton mass matrices

Fukuyama, T. \& Nishiura, H., Model independent constraints of the averaged neutrino masses revisited

Gangopadhyay, G., see Lahiri

Gangopadhyay, S., Saha, A. \& Saha, S., Trace of phase-space noncommutativity in response of a free particle to linearized gravitational waves

Gangopadhyay, S., Voros product and noncommutative inspired black holes

Gao, L. \& Peng, G.-X., Casimir charge and an explanation for $\mathrm{C}$ violation

Gao, T.-J., see Sun

Gao, Y.-H., see Bai

Gao, Z. F., Wang, N., Peng, Q. H., Li, X. D. \& Du, Y. J., Pressure of degenerate and relativistic electrons in a superhigh magnetic field

Garcia i Tormo, X., Review on the determination of $\alpha_{s}$ from the QCD static energy

Gharaei, R., see Golshanian

Ghodsi, O. N. \& Seyyedi, S. A., Influence of breakup process in the fusion reactions with weakly bound nucleus ${ }^{9} \mathrm{Be}$

Ghodsi, O. N. \& Lari, F., The parametrization of fusion barrier characteristics and proximity formalism

Ghodsi, O. N., see Golshanian

Ghose Choudhury, A., see Bagchi

Ghosh, S., see Pramanik

Giachetti, R., see Barducci

Giardino, S. \& Teotônio-Sobrinho, P., A nonassociative quaternion scalar field theory

Giganon, A., see van der Graaf

Giomataris, I., see van der Graaf

Giribet, G., see Babaro

Girotti, H. O., see Ferrari

Gkigkitzis, I., see Haranas

Gogberashvili, M., Sakhelashvili, O. \& Tukhashvili, G., Numerical solutions in 5D standing wave braneworld

Gök, Ç., see Yüksel

Gokbulut, M., Koc, H., Eser, E., Yigitoglu, I. \& Mamedov, B. A., Investigations of the density distri-
28 (2013) 1350146

28 (2013) 1350186

28 (2013) 1350076

28 (2013) 1350161

28 (2013) 1350030

28 (2013) 1350119

28 (2013) 1350060

28 (2013) 1350145

28 (2013) 1350138

28 (2013) 1330028

28 (2013) 1350164

28 (2013) 1350040

28 (2013) 1350116

28 (2013) 1350164

28 (2013) 1375001

28 (2013) 1350038

28 (2013) 1350029

28 (2013) 1350163

28 (2013) 1340021

28 (2013) 1340021

28 (2013) 1330003

28 (2013) 1350052

28 (2013) 1350077

28 (2013) 1350092

28 (2013) 1350177 
butions of ${ }^{208} \mathrm{~Pb}$ with the ThomasFermi method

Gollu, S., Deepthi, K. N. \& Mohanta, R., Charged lepton correction to tri-bimaximal lepton mixing and its implications to neutrino phenomenology

Golshanian, M., Ghodsi, O. N. \& Gharaei, R., Role of surface energy coefficient and temperature of compound nucleus in the $\alpha$-decay process

Gomes, M., see Ferrari

Gong, Y.-G., see Zhang

Gotink, W., see van der Graaf

Grady, M., Toward a proof of long range order in $4 \mathrm{D} \mathrm{SU}(N)$ lattice gauge theory

Granados, V. D., see Martínez

Granda, L. N., Natural scaling for dark energy

Greco, M., see Ermolaev

Greenwald, J., see Moore

Grover, T., Entanglement entropy and strongly correlated topological matter

Gu, J.-A., Mysterious anti-gravity and dark-essence

Gu, P.-H. \& Sarkar, U., Common origin of baryon asymmetry and proton decay

Guha, P., see Bagchi

Gumjudpai, B., Quintessential power-law cosmology: Dark energy equation of state

Gumrukcuoglu, A. E., Lin, C. \& Mukohyama, S., Self-accelerating universe in nonlinear massive gravity

Gunsing, F., see Belloni

Guo, X.-D., see Zhao

Gupta, S. \& Kumar, R., Augmented superfield approach to non-YangMills symmetries of Jackiw-Pi model: Novel observations

Gupta, V., Sánchez-Colón, G. \& Rajpoot, S., Decay coupling constant sum rules for tetraquarks $T[(\bar{Q} q)(Q \bar{q})]$ with broken $\mathrm{SU}(3)$ symmetry

Haba, Z., Relativistic diffusion of quarks in random gluon fields

Hajdukovic, D. S., The signatures of new physics, astrophysics and cosmology?
28 (2013) 1350108

28 (2013) 1350131

28 (2013) 1350164

28(2013) 1350052

28(2013) 1350135

28 (2013) 1340021

28 (2013) 1350087

28(2013) 1350042

28 (2013) 1350117

28 (2013) 1360005

28(2013) 1350055

28 (2013) 1330001

28 (2013) 1340018

28 (2013) 1350159

28 (2013) 1375001

28 (2013) 1350122

28 (2013) 1340016

28 (2013) 1340023

28 (2013) 1350173

28 (2013) 1350011

28 (2013) 1350015

28 (2013) 1350091

28 (2013) 1350124
Halilsoy, M., see Mazharimousavi

Hammad, F., A note on the effect of the cosmological constant on the bending of light

Haranas, I. \& Gkigkitzis, I., Bekenstein bound of information number $N$ and its relation to cosmological parameters in a universe with and without cosmological constant

Harfield, A., see Srisawad

Harikumar, E. \& Verma, R., Uniformly accelerated detector in the $\kappa$-deformed Dirac vacuum

Hartjes, F., see van der Graaf

Hassan, M., see Mushahid

He, X.-G., Li, S.-F. \& Lin, H.-H., Further studies of Higgs properties at an ILC $\gamma \gamma$ collider

Hesari, H. \& Najafabadi, M. M., Probing the anomalous couplings of the top quark with gluon at the LHC and Tevatron

Hessey, N., see van der Graaf

Ho, C. M. \& Weiler, T. J., Physical and stable closed time-like curves

Ho, C. M., Kephart, T. W., Minic, D. \& Ng, Y. J., Spacetime emergence and general covariance transmutation

Høyer, P. \& Rashid, J., Quantum nonlocal boxes exhibit stronger distillability

Hu, C.-Y., Chen, C.-C. \& Chen, P., Cherenkov radiation induced by cosmogenic neutrinos in nearfield

Hu, C.-Y., see Chen

$\mathrm{Hu}, \mathrm{S}$., see Sun

Hu, Y., see Sun

Huang, H.-J., see Ruan

Huang, J.-H. \& Wang, W., Microcausality of spin-induced noncommutative theories

Huang, P., Huang, Y.-C. \& Yuan, F.-F., $f(T)$ gravity from holographic Ricci dark energy model with new boundary conditions

Huang, W., see Sun

Huang, Y.-C., see Huang

Hübsch, T., On supermultiplet twisting and spin-statistics

Hulsbergen, W., Constraining new physics in $B_{s}^{0}$ meson mixing

Ioannou, P. D., see Mazilu

Irastorza, I. G., Ferrer-Ribas, E. \&
28 (2013) 1350073

28 (2013) 1350181

28 (2013) 1350077

28 (2013) 1350070

28 (2013) 1350063

28 (2013) 1340021

28 (2013) 1350020

28 (2013) 1350085

28 (2013) 1350170

28 (2013) 1340021

28 (2013) 1250237

28 (2013) 1350005

28 (2013) 1330012

28 (2013) 1340006

28 (2013) 1340009

28 (2013) 1350114

28(2013) 1350060

28(2013) 1350113

28 (2013) 1250239

28 (2013) 1350171

28 (2013) 1350114

28 (2013) 1350171

28 (2013) 1350147

28 (2013) 1330023

28 (2013) 1350126 
Dafni, T., Micromegas in the rare event searches field

Jahan, A. \& Choudhury, D. K., An analysis of momentum fractions of quarks and gluons in a model of proton

Jamin, M., Strong coupling from tau lepton decays

Jansweijer, P., see van der Graaf

Jawad, A., see Sharif

Jora, R., Schechter, J. \& Shahid, M. N., An analytical treatment of the neutrino masses and mixings

Jora, R. \& Shahid, M. N., Note about the implementation of finite symmetries in the lepton sector

Jung, M., see Chung

Kaloshin, A. E., Kobeleva, E. A. \& Lomov, V. P., Opposite parity fermion mixing and baryons $1 / 2^{ \pm}$

Kamae, T., Fermi - Large area telescope: Accomplishments and challenges

Kansu, M. E., see Tanışlı

Kar, A., Moolekamp, F. \& Rajeev, S. G., A hierarchical finite element method for quantum field theory

Karateev, D., see Ferretti

Karle, A., Neutrino astronomy at the south pole

Kauer, N., Inadequacy of zero-width approximation for a light Higgs boson signal

Kawabe, H., Stochastic quantization approach for causal dynamical triangulation string field theory

Kephart, T. W., see Ho

Khan, K. H., see Ajaz

Khmelnytskaya, K. V., see Rosu

Kile, J., Flavored dark matter: A review

Kim, C.-H., Kim, S. K. \& Park, Y.-J., SU(5/3) superalgebra and its representations of fundamental particles

Kim, H. B., A study on anisotropy in the arrival directions of ultrahigh-energy cosmic rays observed by Pierre Auger observatory

Kim, H.-C., Inflation as an attractor in scalar cosmology

Kim, N., Supersymmetric Wilson loops with general contours in ABJM theory
28 (2013) 1340026

Kim, S. K., see Kim

Kim, Y.-W., Myung, Y. S. \& Park, Y.-J., BRST quantization of a sixth-order derivative scalar field theory

28 (2013) 1350086

28 (2013) 1360006

28 (2013) 1340021

28 (2013) 1350180

28 (2013) 1350184

28 (2013) 1350185

28 (2013) 1350100

28 (2013) 1350156

28 (2013) 1340002

28 (2013) 1350026

28 (2013) 1350139

28 (2013) 1350025

28 (2013) 1340004

28 (2013) 1330015

28 (2013) 1350013

28 (2013) 1350005

28 (2013) 1350175

28 (2013) 1340017

28 (2013) 1330031

28 (2013) 1350075

28 (2013) 1350089

28 (2013) 1350150
Kim, Y.-W., see Choi

Kisslinger, L. S. \& Das, D., Upsilon production in $p p$ collisions for forward rapidities at LHC

Kisslinger, L. S. \& Das, D., $\Psi$ and $\Upsilon$ production in $p p$ collisions at 7.0 TeV

Kisslinger, L. S., Review of recent neutrino physics research

Kisslinger, L. S., Neutrino energies in a neutrinosphere

Kitabayashi, T., Bipair neutrino mixing and leptogenesis

Klein, M., see Brüning

Klimenko, K. G., Zhokhov, R. N. \& Zhukovsky, V. Ch., Superconductivity phenomenon induced by external in-plane magnetic field in $(2+1)$-dimensional Gross-Neveu type model

Klinkhamer, F. R., Neutrino mass and the standard model

Klinkhamer, F. R., Black-hole solution without curvature singularity

Kniehl, B. A., see Butenschoen

Kobeleva, E. A., see Kaloshin

Koc, H., see Gokbulut

Konig, A., see van der Graaf

Koppert, W., see van der Graaf

Kou, L.-N., see Sun

Kouwn, S., Lee, J., Lee, T. H. \& Oh, P., ELKO spinor model with torsion and cosmology

Kowalski-Glikman, J. \& Rosati, G., Relative locality in curved spacetime

Król, J., see Asselmeyer-Maluga

Kruglov, S. I., Modified wave equation for spinless particles and its solutions in an external magnetic field

Kühn, J. H., Precise heavy quark masses

Kumar, L., Review of recent results from the RHIC beam energy scan Kumar, R., see Gupta

Kuriakose, V. C., see Tharanath

Kuriakose, V. C., see Sebastian

Kürkçüoğlu, S., see Balachandran

Kusenko, A., Cosmic connections:
28 (2013) 1350084

28 (2013) 1350182

28 (2013) 1350143

28 (2013) 1350067

28 (2013) 1350120

28 (2013) 1330024

28 (2013) 1350153

28 (2013) 1350016

28 (2013) 1330011

28 (2013) 1350096

28 (2013) 1350010

28 (2013) 1350136

28 (2013) 1350027

28 (2013) 1350156

28 (2013) 1350108

28 (2013) 1340021

28 (2013) 1340021

28 (2013) 1350060

28 (2013) 1350121

28 (2013) 1350101

28 (2013) 1350158

28 (2013) 1350014

28 (2013) 1360019

28 (2013) 1330033

28 (2013) 1350011

28 (2013) 1350003

28 (2013) 1350149

28 (2013) 1350028 
From cosmic rays to gamma rays, cosmic backgrounds and magnetic fields

Labun, L., see Rafelski

Labun, L. \& Rafelski, J., Temperature of electron fluctuations in an accelerated vacuum

Lafferty, G. D., Tau lepton hadronic decays at BABAR and BELLE

Lahiri, C. \& Gangopadhyay, G., Importance of Q-values in astrophysical rapid proton capture process

Lai, K.-C., see Chen

Lai, K.-C., see Liu

Lampe, B., Color and isospin waves from tetrahedral Shubnikov groups

Lari, F., see Ghodsi

Lee, J., see Kouwn

Lee, T. H., see Kouwn

Leutwyler, H., The mass of the two lightest quarks

Li, G.-L., see Zhao

Li, H., see Peng

Li, H., see Zhang

Li, S.-F., see $\mathrm{He}$

Li, X. D., see Gao

Li, Y.-N., see Ruan

Li, Y.-Y., Motions of Cartan curves in $n$-dimensional Minkowski space

Liang, S., see Tao

Limphirat, A., see Srisawad

Lin, C., see Gumrukcuoglu

Lin, G.-L., see Liu

Lin, H.-H., see He

Ling, Y. \& Pan, W.-J., $(m, n)$-type holographic dark energy models

Liu, L.-X., see Cornell

Liu, T. C., Lai, K.-C. \& Lin, G.-L., Neutrino flavor ratios on earth under decay and oscillation scenarios

Llopart, X., see van der Graaf

Lomov, V. P., see Kaloshin

Ludwick, K. J., see Frampton

Luo, G.-H., see Sun

Luo, L.-J., Shi, S. \& Zong, H.-S., The study of QCD phase transition at finite temperature and chiral chemical potential in a DysonSchwinger equation model

Luongo, O., Dark energy from a positive jerk parameter

Ma, B.-Q., New perspective on space
28 (2013) 1340001

28 (2013) 1340014

28 (2013) 1340015

28 (2013) 1360007

28 (2013) 1350076

28(2013) 1340009

28(2013) 1340005

28(2013) 1350018

28 (2013) 1350116

28 (2013) 1350121

28(2013) 1350121

28 (2013) 1360014

28(2013) 1350129

28 (2013) 1350133

28 (2013) 1350009

28 (2013) 1350085

28 (2013) 1350138

28(2013) 1350113

28 (2013) 1350110

28 (2013) 1350081

28 (2013) 1350070

28 (2013) 1340016

28 (2013) 1340005

28 (2013) 1350085

28 (2013) 1350128

28(2013) 1330007

28 (2013) 1340005

28 (2013) 1340021

28(2013) 1350156

28 (2013) 1350125

28 (2013) 1350151

28 (2013) 1350105

28 (2013) 1350080

28 (2013) 1340012; and time from Lorentz violation

Ma, M.-S., see Zhao

1392001

28 (2013) 1350129

Maas, A., Bound-state/elementaryparticle duality in the Higgs sector and the case for an excited "Higgs" within the Standard Model

Maiella, G., see Cristofano

Maintas, X. N., see Mazilu

Malaescu, B., Hadronic $\tau$ and $e^{+} e^{-}$ spectra, contribution to $(g-2)_{\mu}$ and QCD studies

Mamedov, B. A., see Gokbulut

Mart, T. \& Sari, A. K., Hadronic form factors in the $\gamma p \rightarrow K^{+} \Lambda$ process

Martínez, D., Salazar-Ramírez, M., Mota, R. D. \& Granados, V. D., On the supersymmetry of the DiracKepler problem plus a Coulombtype scalar potential in $(D+1)$ dimensions and the generalized Lippmann-Johnson operator

Martynov, M. V., see Frolov

Mavromatos, N. E., Sarkar, S. \& Tarantino, W., Condensate structure of D-brane defect induced flavor vacuum

Mazharimousavi, S. H. \& Halilsoy, M., Rindler type acceleration in $f(R)$ gravity

Mazilu, N., Ioannou, P. D., Diakonos, F. K., Maintas, X. N. \& Agop, M., A quark-independent description of confinement

McNeile, C., Strong coupling and quark masses from lattice QCD

\section{Mei, H.-H., see $\mathrm{Ni}$}

Meng, X.-W., see Tao

Merad, M., see Benzair

Mikhailov, A., Schwarz, A. \& Xu, R., Cohomology ring of the BRST operator associated to the sum of two pure spinors

Minic, D., see Ho

Minkevich, A. V., On theory of regular accelerating universe in Riemann-Cartan spacetime

Minkowski, P., Light quark mass ratios $\left(m_{u}: m_{d}: m_{s}\right)$ from meson and baryon mass splittings

Moazzen, M., see Tofighi

Moch, S.-O., see Alekhin

Mohanta, R., see Gollu

Mohanty, S., see Chakrabortty

Montani, G., see Pugliese

Moolekamp, F., see Kar
28 (2013) 1350103

28 (2013) 1350066

28 (2013) 1350126

28 (2013) 1360011

28 (2013) 1350108

28 (2013) 1350054

28 (2013) 1350042

28 (2013) 1350035

28 (2013) 1350045

28 (2013) 1350073

$28(2013) 1350126$

28 (2013) 1360012

28 (2013) 1340013

28 (2013) 1350081

28 (2013) 1350144

28 (2013) 1350107

28 (2013) 1350005

28 (2013) 1350090

28 (2013) 1360015

28 (2013) 1350044

28 (2013) 1360018

28 (2013) 1350131

28 (2013) 1350032

28 (2013) 1330013

28 (2013) 1350139 
Moore, D., Greenwald, J. \& Cleaver, G. Gauge models in $D$ dimensions

Mota, R. D., see Martínez

Motavalli, H., see Zeynali

Muhammad, A., see Tao

Mukohyama, S., see Gumrukcuoglu

Mushahid, N., Hassan, M. \& Saleem, U., Conserved quantities in the generalized Heisenberg magnet (GHM) model

Mushahid, N. \& ul Hassan, M., On the dressing method for the generalized coupled dispersionless integrable system

Mustafa, O., Auxiliary quantization constraints on the Von Roos ordering-ambiguity at zero binding energies; azimuthally symmetrized cylindrical coordinates

Myung, Y. S., see Kim

Najafabadi, M. M., see Hesari

Nakayama, Y., $(0,2)$ chiral Liouville field theory

Nam, J. et al. (For the UFFO Collab.), The UFFO slewing mirror telescope for early optical observation from gamma ray bursts

Nemes, M. C., see Felipe

$\mathrm{Ng}$, Y. J., see Ho

Ni, W.-T., Mei, H.-H. \& Wu, S.-J., Foundations of classical electrodynamics, equivalence principle and cosmic interactions: A short exposition and an update

Ni, W.-T., see Wang

Nishiura, H., see Fukuyama

Nishiura, H., see Fukuyama

Nishiura, H., see Fukuyama

Nomura, D., see Cho

Odaka, S., Precise simulation of the initial-state QCD activity associated with $Z$-boson production in hadron collisions

Oh, J. J. \& Yang, H. S., Einstein manifolds as Yang-Mills instantons

Oh, P., see Kouwn

Ohno, Y., see Cho

Onofrio, R., On weak interactions as short-distance manifestations of gravity

Oswald, C. \& Pedlar, T. K., Results in $B_{s}$ physics and bottomonium spectroscopy using the Belle $\Upsilon(5 \mathrm{~S})$ data
28 (2013) 1350055

28 (2013) 1350042

28 (2013) 1350047

28 (2013) 1350081

28 (2013) 1340016

28 (2013) 1350020

$\mathbf{8}(2013) 1350088$

28 (2013) 1350082

28 (2013) 1350182

28 (2013) 1350170

28 (2013) 1350178

28 (2013) 1340003

28 (2013) 1350078

28 (2013) 1350005

28 (2013) 1340013

28 (2013) 1350094

28 (2013) 1350017

28 (2013) 1350146

28 (2013) 1350186

28 (2013) 1350148

(2013) 1350098

28 (2013) 1350097

28 (2013) 1350121

28 (2013) 1350148

28 (2013) 1350022

28 (2013) 1330036
Oudih, M. R., Fellah, M., Allal, N. H. \& Benhamouda, N., Microscopic analysis of shape evolution in Mo isotopes

Pahlavani, M. R., Alavi, S. A. \& Tahanipour, N., Effect of nuclear deformation on the potential barrier and alpha-decay half-lives of superheavy nuclei

Palazzo, A., Phenomenology of light sterile neutrinos: A brief review

Pan, W.-J., see Ling

Papaevangelou, T., see Belloni

Papini, G., Fermion-antifermion mixing in gravitational fields

Park, Y.-J., see Choi

Park, Y.-J., see Kim

Park, Y.-J., see Kim

Parwani, R. R., see Ching

Pascu, G., see Cotăescu

Pavšič, M., Stable self-interacting Pais-Uhlenbeck oscillator

Pedlar, T. K., see Oswald

Peng, G.-X., see Gao

Peng, Q. H., see Gao

Peng, R. \& Li, H., Fragmentation functions of gluon splitting into charmed hadrons in the recombination model

Pholwiang, S., see Srisawad

Piattella, O. F., see Fabris

Pilaftsis, A., Anomalous fermion mass generation at three loops

Pinto, S. D., GEM applications outside high energy physics

Plessas, W., Effective degrees of freedom in low-energy quantum chromodynamics

Popovici, C., Dyson-Schwinger approach to strongly coupled theories

Posolda, P., Study of ambiguities of QCD predictions at jet production in proton-proton collisions at next-to-leading order

Pramanik, S. \& Ghosh, S., On the stability of coherent states for PaisUhlenbeck oscillator

Preface

Procureur, S., Micromegas trackers for hadronic physics

Pronskikh, V. S., Radiation studies for the Mu2e experiment: A review

Prudêncio, T. \& Cirilo-Lombardo, D. J., Coherent states, superposi-
28 (2013) 1350134

28 (2013) 1350065

28 (2013) 1330004

28 (2013) 1350128

28 (2013) 1340023

28 (2013) 1350071

28 (2013) 1350143

28 (2013) 1350084

28 (2013) 1350182

28 (2013) 1350061

28 (2013) 1350160

28 (2013) 1350165

28 (2013) 1330036

28 (2013) 1350119

28 (2013) 1350138

28 (2013) 1350133

28 (2013) 1350070

28 (2013) 1350169

28 (2013) 1350083

28 (2013) 1340025

28 (2013) 1360022

28 (2013) 1330006

28 (2013) 1350050

28 (2013) 1350038

28 (2013) 1302001

28 (2013) 1340024

28 (2013) 1330014 
tions of coherent states and uncertainty relations on a Möbius strip

Pugliese, D. \& Montani, G., Astrophysical evidence for an extra dimension: Phenomenology of a Kaluza-Klein theory

Qi, B.-G., see Bai

Rabochaya, Y., see Brevik

Rafelski, J. \& Labun, L., Critical acceleration and quantum vacuum

Rafelski, J., see Labun

Rafelski, J., see Birrell

Rahman, A., see Ajaz

Rajeev, S. G., see Kar

Rajpoot, S., see Gupta

Rani, S., see Sharif

Rashed, A. \& Datta, A., The 2-3 symmetry and large reactor mixing angle

Rashid, J., see Høyer

Raut, S. K., Effect of nonzero $\theta_{13}$ on the measurement of $\theta_{23}$

Ricciardi, G., Determination of the CKM matrix elements $\left|V_{x b}\right|$

Richarte, M. G., see Chimento

Richarte, M. G., see Chimento

Robens, T., Nagy-Soper subtraction: A review

Robertson, R. G. H., Empirical survey of neutrinoless double beta decay matrix elements

Rodríguez, Y., see Beltrán Almeida

Romero, J. M., Escobar-Aguilar, E. S. \& Vázquez, E., Free particle in very special relativity, gauge symmetry and two-time physics

Ropelewski, L., see Titov

Rosati, G., see Kowalski-Glikman

Rosu, H. C. \& Khmelnytskaya, K. V., Inhomogeneous barotropic FRW cosmologies in conformal time

Roszkowski, L., Sessolo, E. M. \& Tsai, Y.-L. S., Bayesian implications of collider and SUSY dark matter direct and indirect searches

Rothkopf, A., From complex to stochastic potential: Heavy quarkonia in the quark-gluon plasma

Rövekamp, J., see van der Graaf

Roy, P., see Aizawa

Ruan, D., Huang, H.-J. \& Li, Y.-N., Single boson and inversion boson realizations of multi-parameter nonlinearly deformed angular momentum algebra
28 (2013) 1350058

28 (2013) 1330013

28 (2013) 1350145

28(2013) 1350172

28 (2013) 1340014

28 (2013) 1340015

28 (2013) 1350188

28(2013) 1350175

28 (2013) 1350139

28 (2013) 1350015

28 (2013) 1350118

28 (2013) 1330030

28(2013) 1330012

28 (2013) 1350093

28 (2013) 1330016

28 (2013) 1250235

28 (2013) 1250236

$28(2013) 1330020$

28 (2013) 1350021

28 (2013) 1350012

28 (2013) 1350004

28 (2013) 1340022

28(2013) 1350101

$28(2013) 1340017$

28 (2013) 1340008

28 (2013) 1330005

28 (2013) 1340021

28 (2013) 1350123

28 (2013) 1350113
Rudra, P., Role of generalized cosmic Chaplygin gas in accelerating universe: A field theoretical prescription

Rusek, K., see Aygun

Saha, A., see Gangopadhyay

Saha, S., see Gangopadhyay

Sakalli, I., Quasinormal modes of charged dilaton black holes and their entropy spectra

Sakhelashvili, O., see Gogberashvili

Sakhi, Z., see Zarrouki

Salako, I. G., see Fabris

Salazar-Ramírez, M., see Martínez

Saleem, R., see Sharif

Saleem, U., see Mushahid

Salm, C., see van der Graaf

Sampaio, M., see Felipe

Sánchez, I. E., see Chimento

Sánchez-Colón, G., see Gupta

Sánchez-Delgado, J. M., see Calderón Martín

Sánchez-Vega, B. L. \& Shapiro, I. L., The case of asymptotic supersymmetry

Sannino, F., Jumping dynamics

Sannino, F., see Antipin

Santillán, O. P., A common scenario leading to a small vacuum energy and stable super-massive particles

Santos, A. F., see Ulhoa

Santos, A. F., Gödel solution in $f(R, T)$ gravity

Sari, A. K., see Mart

Sarkar, S., see Mavromatos

Sarkar, U., see $\mathrm{Gu}$

Sarmadmasood, S., see Tao

Sato, M. \& Tsuchiya, A., Note on consistent truncation over higherdimensional sphere in supergravity

Savickas, D., Kinematic repulsions between inertial systems in an expanding inflationary universe

Schechter, J., see Jora

Schilcher, K., Light quark masses from QCD sum rules

Schimmrigk, R., Automorphic black hole entropy

Schimmrigk, R., Motivic $L$-function identities from CFT and arithmetic mirror symmetry

Schmitz, J., see van der Graaf

Schwarz, A., see Mikhailov

Scomparin, E., Charmonium pro-
28 (2013) 1350102

28 (2013) 1350112

28 (2013) 1350161

28 (2013) 1350161

28 (2013) 1350109

28 (2013) 1350092

28 (2013) 1350062

28 (2013) 1350169

28 (2013) 1350042

28 (2013) 1350072

28 (2013) 1350020

28 (2013) 1340021

28 (2013) 1350078

28 (2013) 1250236

28 (2013) 1350015

28 (2013) 1350008

28 (2013) 1350053

28 (2013) 1350127

28 (2013) 1350140

28 (2013) 1350099

28 (2013) 1350039

28 (2013) 1350141

28 (2013) 1350054

28 (2013) 1350045

28 (2013) 1350159

28 (2013) 1350081

28 (2013) 1350104

28 (2013) 1330025

28 (2013) 1350184

$28(2013) 1360016$

28 (2013) 1330026

28 (2013) 1350167

28 (2013) 1340021

28 (2013) 1350107 
duction in $\mathrm{Pb}-\mathrm{Pb}$ collisions at ALICE: From suppression to regeneration?

Sebastian, S. \& Kuriakose, V. C., Spectroscopy and thermodynamics of MSW black hole

Şengör, G. \& Arık, M., A fivedimensional model with a fifth dimension as fundamental as time in terms of a cosmological approach

Senol, A., see Çakir

Sert, Ö., Electromagnetic duality and new solutions of the nonminimally coupled $Y(R)$-Maxwell gravity

Sert, Ö. \& Adak, M., Non-minimal $R F^{2}$-type corrections to holographic superconductor

Sessolo, E. M., see Roszkowski

Seyyedi, S. A., see Ghodsi

Shahid, M. N., see Jora

Shahid, M. N., see Jora

Shapiro, I. L., see Sánchez-Vega

Sharif, M. \& Jawad, A., Interacting generalized dark energy and reconstruction of scalar field models

Sharif, M. \& Rani, S., Viscous dark energy in $f(T)$ gravity

Sharif, M. \& Saleem, R., Thermodynamics in Kaluza-Klein universe

Sharif, M. \& Zahra, Z., Static plane symmetric interior solutions in $f(R)$ gravity

Shchigolev, V. K., Fractional Einstein-Hilbert action cosmology

Shen, Y.-Q., see Tao

Shi, S., Sun, W.-M. \& Zong, H.-S., Chiral anomaly of massless fermion at finite temperature and chemical potential

Shi, S., see Luo

Shi, Z.-Q., Left-right polarization asymmetry of the weak interaction mass of polarized fermions in flight

Shifman, M., Remarks on adjoint QCD with $k$ flavors, $k \geq 2$

Shojaei-Fard, A., The global $\beta$ functions from solutions of Dyson-Schwinger equations

Singh, N. K., Unimodular constraint on global scale invariance

Slad, L. M., Magnetic moment operator of non-Dirac particles and some elements of polarization $e p$ experiments

Smirnov, A. D., see Frolov

Smits, S., see van der Graaf

28 (2013) 1330018

Spiesberger, H., An operator product expansion analysis of $e^{+} e^{-}$annihilation data

28 (2013) 1350149

28 (2013) 1350095

28 (2013) 1350142

28 (2013) 1350049

28 (2013) 1350190

28 (2013) 1340008

28 (2013) 1350040

28 (2013) 1350184

28 (2013) 1350185

28 (2013) 1350053

28 (2013) 1350180

28 (2013) 1350118

28 (2013) 1350072

28 (2013) 1350041

28 (2013) 1350056

28 (2013) 1350081

28 (2013) 1350006

28 (2013) 1350105

28 (2013) 1350059

28 (2013) 1350179

28 (2013) 1350152

28 (2013) 1350130

28 (2013) 1350051
Spousta, M., Jet quenching at LHC

Srisawad, P., Suksri, A., Pholwiang, S., Harfield, A., Zheng, Y.-M., Yan, Y. P. \& Limphirat, A., Transverse mass spectra and rapidity distributions of $\mathrm{K}^{+}$in Ni-Ni collisions at $1.93 \mathrm{~A} \mathrm{GeV}$

Stojkovic, D., Vanishing dimensions: A review

Stornaiolo, C., see Cristofano

Suksri, A., see Srisawad

Suleymanov, M. K., see Ajaz

Sun, F., Feng, T.-F., Zhao, S.-M., Gao, T.-J., Kou, L.-N., Sun, K.-S. $\& \mathrm{Hu}, \mathrm{Y}$., $B^{0}-\bar{B}^{0}$ mixing with the constraint of a $125 \mathrm{GeV}$ Higgs

Sun, K.-S., see Sun

Sun, K.-S., Feng, T.-F., Luo, G.-H., Yang, X.-Y. \& Chen, J.-B., Lepton flavor violation in inverse seesaw model

Sun, L.-F., Yan, M.-L., Deng, Y., Huang, W. \& Hu, S., Schwarzschild-de Sitter metric and inertial Beltrami coordinates

Sun, W.-M., see Shi

Sun, W.-M., see Wang

Tahanipour, N., see Pahlavani

Tang, Y., Vacuum stability in the standard model

Tanışlı, M., Kansu, M. E. \& Demir, S., Supersymmetric quantum mechanics and Euclidean-Dirac operator with complexified quaternions

Tao, J.-Q., Shen, Y.-Q., Fan, J.-W., Xiao, H., Chen, G.-M., Chen, H.S., Bian, J.-G., Chen, Y., Liang, S., Meng, X.-W., Muhammad, A., Sarmadmasood, S., Wang, Z. \& Zhu, S.-H., Effect from the gluonfusion signal and background interference for Higgs decaying to $\gamma \gamma$ analysis at the LHC

Tarantino, W., see Mavromatos Tarhini, A., see Cornell

Tasci, A. T., see Çakir

Teotônio-Sobrinho, P., see Giardino

Tharanath, R. \& Kuriakose, V. C., Thermodynamics and spectroscopy of Schwarzschild black hole
28 (2013) 1350035

28 (2013) 1340021

28 (2013) 1360009

28 (2013) 1330017

28 (2013) 1350070

28 (2013) 1330034

28 (2013) 1350066

28 (2013) 1350070

28(2013) 1350175

28 (2013) 1350060

28 (2013) 1350060

28 (2013) 1350151

28 (2013) 1350114

28 (2013) 1350006

28 (2013) 1350064

28 (2013) 1350065

28 (2013) 1330002

28 (2013) 1350026

28 (2013) 1350081

28 (2013) 1350045

28 (2013) 1330007

28 (2013) 1350142

28 (2013) 1350163 
surrounded by quintessence Timmermans, J., see van der Graaf Timoshkin, A. V., see Brevik

Titov, M. \& Ropelewski, L., Micropattern gaseous detector technologies and RD51 Collaboration

Tofighi, A. \& Moazzen, M., Stabilization of modulus in RandallSundrum model I by bulk scalar fields

Tossa, J., see Fabris

Troshin, S. M. \& Tyurin, N. E., On the double-ridge effect at the LHC

Troyan, S. I., see Ermolaev

Tsai, Y.-L. S., see Roszkowski

Tsuchiya, A., see Sato

Tukhashvili, G., see Gogberashvili

Tunstall, L. C., see Crewther

Tuominen, K., see Antipin

Tyurin, N. E., see Troshin

ul Hassan, M., see Mushahid

Ulhoa, S. C., Santos, A. F. \& Amorim, R. G. G., On the energymomentum flux in Gödel-type models

Valenzuela-Toledo, C. A., see Beltrán Almeida

van Bakel, N., see van der Graaf van Beuzekom, M., see van der Graaf van der Graaf, H., Aarnink, T., Aarts, A., van Bakel, N., Berbee, E., Berkien, A., van Beuzekom, M., Bosma, M., Campbell, M. Chefdeville, M., Colas, P., Colijn, A.-P., Fornaini, A., Fransen, M., Giganon, A., Giomataris, I., Gotink, W., de Groot, N., Hartjes, F., van der Heijden, B., Hessey, N., Jansweijer, P., Konig, A., Koppert, W., Llopart, X., de Nooij, L., van der Putten, S., Rövekamp, J., Salm, C., Bello, D. S. S., Schmitz, J., Smits, S., Timmermans, J., Verkooijen, H., Visschers, J., Visser, J., Wijnen, T. \& Wyrsch, N., The gridpix detector: History and perspective

van der Heijden, B., see van der Graaf

van der Putten, S., see van der Graaf Vázquez, E., see Romero

Velten, H. E. S., see Fabris

Venkataratnam, K. K., Behavior of non-classical inflaton in the FRW universe

Verkooijen, H., see van der Graaf
28(2013) 1350003 28 (2013) 1340021

28(2013) 1350172

28 (2013) 1340022

28 (2013) 1350044 28 (2013) 1350169

28 (2013) 1350031 28 (2013) 1360005 28 (2013) 1340008 28(2013) 1350104 28(2013) 1350092 28 (2013) 1360010 28 (2013) 1350140 28 (2013) 135003 28 (2013) 1350088

28 (2013) 1350039

28 (2013) 1350012 28 (2013) 1340021 28 (2013) 1340021

28(2013) 1340021

28 (2013) 1340021

28 (2013) 1340021

28 (2013) 1350004

28 (2013) 1350169

28 (2013) 1350168 28(2013) 1340021
Verma, R., see Harikumar

Vignolo, S., see Capozziello

Visinelli, L., Axion-electromagnetic waves

Visschers, J., see van der Graaf

Visser, J., see van der Graaf

Wagner, O. E., Dark matter density as a function of the time of year

Wallis, D. H., see Wickramasinghe

Wallis, J., see Wickramasinghe

Wang, B., Sun, W.-M. \& Zong, H.-S., The chiral susceptibility around the critical end point

Wang, C.-H., see Chen

Wang, F., see Zhao

Wang, L.-B. \& Ni, W.-T., Proton radius puzzle and large extra dimensions

Wang, N. \& Xu, L., Strong gravitational lensing and its cosmic constraints

Wang, N., see Gao

Wang, S., see Zhong

Wang, W., see Huang

Wang, Z., see Tao

Ward, B. F. L., IR-improved operator product expansions in nonAbelian gauge theory

Weiler, T. J., see Ho

Wickramasinghe, N. C., Wallis, J. \& Wallis, D. H., PANSPERMIA: Evidence from astronomy to meteorites

Wijnen, T., see van der Graaf

Wittig, H., Low-energy QCD II Status of lattice calculations

Wong, H. T., see Yue

Wotschack, J., The development of large-area micromegas detectors for the ATLAS upgrade

$\mathrm{Wu}, \mathrm{S} .-\mathrm{J}$. , see $\mathrm{Ni}$

Wu, Y.-L., see Cui

Wyrsch, N., see van der Graaf

$\mathrm{Xiao}, \mathrm{H}$., see Tao

Xiao, Y. \& Yang, G., A realization of the holographic entropy

$\mathrm{Xu}, \mathrm{L}$., see Wang

$\mathrm{Xu}, \mathrm{R}$., see Mikhailov

$\mathrm{Xu}, \mathrm{W}$., see Zhao

$\mathrm{Xu}, \mathrm{X} .-\mathrm{B}$. , see Bai

Yan, M.-L., see Sun

Yan, Y. P., see Srisawad

Yang, C.-T., see Birrell

Yang, G., see Xiao

Yang, H. S., see $\mathrm{Oh}$

Yang, X.-Y., see Sun
28 (2013) 1350063

28 (2013) 1350155

28 (2013) 1350162

28 (2013) 1340021

28 (2013) 1340021

28 (2013) 1330022

28 (2013) 1330009

28 (2013) 1330009

28 (2013) 1350064

28 (2013) 1340010

28 (2013) 1350173

28 (2013) 1350094

28 (2013) 1350057

28 (2013) 1350138

28 (2013) 1350043

28 (2013) 1250239

28 (2013) 1350081

28 (2013) 1350069

28 (2013) 1250237

28 (2013) 1330009

28 (2013) 1340021

28 (2013) 1360013

28 (2013) 1340007

28 (2013) 1340020

28 (2013) 1340013

28 (2013) 1350132

28 (2013) 1340021

28 (2013) 1350081

28 (2013) 1350046

28 (2013) 1350057

28 (2013) 1350107

28 (2013) 1350002

28 (2013) 1350145

28 (2013) 1350114

28 (2013) 1350070

28 (2013) 1350188

28 (2013) 1350046

28 (2013) 1350097

28 (2013) 1350151 
Ydri, B. \& Bouchareb, A., On the problem of vacuum energy in FLRW universes and dark energy

Yigitoglu, I., see Gokbulut

Yilmaz, A. H., see Bayram

Younis, H., see Ajaz

Yu, P., see Zhao

Yu, Y. \& Zheng, S., Oblique corrections in the MSSM at one loop. II. fermions

Yu. Petrov, A., see Ferrari

Yuan, F.-F., see Huang

Yue, Q. \& Wong, H. T., Dark matter search with sub-keV Germanium detectors at the China Jinping underground laboratory

Yüksel, E., Gök, Ç \& Bozkurt, K., Detailed investigation of the tensor force on the evaluation of the single particle levels of $Z=28$ and $Z=82$ nuclei

Zahra, Z., see Sharif

Zaman, A., see Ajaz

Zarrouki, R., Sakhi, Z. \& Bennai, M., Tachyonic braneworld inflation and observations

Zaslavskii, O. B., Acceleration of particles as a universal property of ergosphere

Zeng, M., see Zhong

Zeng, Y., Precise measurement of the $W$-boson mass at $\mathrm{CDF}$

Zeynali, K., Darabi, F. \& Motavalli, H., Multi-dimensional cosmology and DSR-GUP

Zhan, X.-J., see Zhao

Zhang, H.-B., see Zhao

Zhang, L., Li, H., Zhao, R. \& Cai, R.,
The entropy of a dielectric black hole

28 (2013) 1350166

28 (2013) 1350108

28 (2013) 1350068

28 (2013) 1350175

28 (2013) 1350002

28 (2013) 1250238

28 (2013) 1350052

28 (2013) 1350171

28 (2013) 1340007

28 (2013) 1350177

28 (2013) 1350041

28 (2013) 1350175

28 (2013) 1350062

28 (2013) 1350037

28 (2013) 1350043

28 (2013) 1330019

28 (2013) 1350047

28 (2013) 1350173

28 (2013) 1350173
Zhang, L.-C., see Zhao

Zhang, Y. \& Gong, Y.-G., The "coincidence" problem in modified holographic dark energy model

Zhao, H.-H., Li, G.-L., Zhao, R., Ma, M.-S. \& Zhang, L.-C., Entanglement entropy of $d$-dimensional black hole and quantum isolated horizon

Zhao, L., Yu, P. \& Xu, W., Hamiltonian description of singular Lagrangian systems with spontaneously broken time translation symmetry

Zhao, R., see Zhang

Zhao, R., see Zhao

Zhao, S.-M., see Sun

Zhao, S.-M., Wang, F., Chen, B., Feng, T.-F., Zhang, H.-B., Guo, X.-D. \& Zhan, X.-J., Some twoloop contributions to muon magnetic dipole moment in the $\mathrm{CP}$ violating MSSM

Zheng, S., see $\mathrm{Yu}$

Zheng, Y.-M., see Srisawad

Zhokhov, R. N., see Klimenko

Zhong, P., Wang, S. \& Zeng, M., Some exact blowup solutions to multidimensional Schrödinger map equation on hyperbolic space and cone

Zhu, S.-H., see Tao

Zhukovsky, V. Ch., see Klimenko

Zong, H.-S., see Luo

Zong, H.-S., see Shi

Zong, H.-S., see Wang
28 (2013) 1350009

28 (2013) 1350129

28 (2013) 1350135

28 (2013) 1350129

28 (2013) 1350002

28 (2013) 1350009

28 (2013) 1350129

28 (2013) 1350060

28 (2013) 1350173

28 (2013) 1250238

28 (2013) 1350070

28 (2013) 1350096

28 (2013) 1350043

28 (2013) 1350081

28 (2013) 1350096

28 (2013) 1350105

28 (2013) 1350006

28 (2013) 1350064 